Ann. Abeille, I966, 9 (I), 55-65.

\title{
LES INSECTES ANTHOPHILES DE ROUMANIE
}

\author{
E. SANDULÉAC \\ Bucarest-Kitila (Roumanie)
}

SOMMAIRE

\begin{abstract}
L'article présente une synthèse sur le comportement pollinisateur des Apoïdea de la région carpatho-danubienne, l'adaptation des Apoïdea à l'anthophilie et un inventaire des pollinisateurs de la région carpatho-danubienne. Ce travail constitue un tour d'horizon sur nos connaissances actuelles des relations entre insectes et fleurs.
\end{abstract}

\section{INTRODUCTION}

Certains insectes visitent les fleurs pour rechercher leur nourriture ou celle de leur progéniture, ou bien pour copuler, dormir ou s'abriter. Quelques-uns déposent leurs œufs sur l'ovaire de la fleur afin que la larve puisse en consommer le fruit. Les parasites - imagos ou larves - recherchent leurs hôtes sur les fleurs. La plupart des insectes anthophiles sont attirés par le nectar et le pollen. Tout en s'alimentant, ils transportent le pollen d'une fleur sur l'autre, réalisant la pollinisation croisée et permettant ainsi la survivance des plantes. Les Diptères et les Coléoptères visitent principalement les fleurs à corolle plate, où la source de nectar est en surface. Les insectes pourvus d'un long proboscis, tels qu'Hyménoptères et Lépidoptères vont chercher le nectar dans les fleurs à corolle profonde.

Depuis le milieu du xvirr ${ }^{\mathrm{e}}$ siècle, les botanistes ont étudié les relations étroites qui existent entre fleurs et insectes ; mais ce n'est qu'après la publication, en I859, de l'ouvrage de DARWin, dans lequel est soulignée l'importance de la pollinisation croisée, que les recherches concernant l'évolution concomitante des fleurs et des insectes anthophiles ont été intensifiées. Les connaissances de l'époque furent rassemblées dans 1'ouvrage de MÜLLER (I873) et, plus tard, dans celui de HEINECK (I929), Grant (r949) a traité également certains aspects des relations entre fleurs et insectes, signalant le rôle des abeilles dans l'évolution et la spécification des Angiospermes. 


\section{ADAPTATION DES APOÏDEA A I'ANTHOPHILIE}

Dès le début du xix ${ }^{\mathrm{e}}$ siècle, un grand nombre d'entomologistes ont étudié les corrélations étroites entre les Apoïdes et les fleurs. On peut trouver de nombreuses références ainsi que des observations personnelles dans les publications de FRIESE (I923) et WHEELER (I926). MALISHEV (I933) a complété beaucoup de ses publications sur la biologie des abeilles en fournissant des méthodes de recherche et d'étude des nids d'Apoïdea, et, en I957, il a soutenu que l'apparition et l'évolution des Angiospermes ont été dues à un développement rapide et une large dissémination de ces Hyménoptères anthophiles. Michener, dans ses nombreux travaux sur les abeilles (I953, I956, I958), a donné d'amples informations et fait un exposé critique. En I958, LINSLEY a fait paraître un résumé utile à ceux qui étudient la vie des Apoïdea : c'est une interprétation pertinente des résultats acquis pendant ${ }_{5} 5^{\circ}$ années d'observations. De nombreuses études et expérimentations ont été entreprises, principalement en U. R. S. S., dans le but d'accroître la production de fruits, légumes ou graines au moyen de la pollinisation entomophile, aussi bien en ce qui concerne 1'abeille (Davidova, I948, I956 ; Filatov, I945 ; Komarov, I956 ; Oresov, I956 ; Schibrea, I947, etc.) que les autres Apoïdea (Blagoveshchenskaia, I954 ; JuraVLEV, I956; PANfiLov, I956, etc.). La transformation des plantes anémophiles en plantes entomophiles par des espèces anciennes de pollinisateurs a été mise en évidence chez les Chenopodiaceae en I952, par Popov. Fin I956, le même auteur faisait remarquer que, bien que l'évolution des Angiospermes ait eu lieu parallèlement et liée à celle de leurs agents pollinisateurs, les caractères monolectiques, oligolectiques $\left({ }^{1}\right)$ et principalement polylectiques des femelles d'Apoïdea sont relativement instables. Goubine et KILALIFMAN (I958) ont fait une bonne analyse de nos connaissances actuelles sur les relations entre abeilles et fleurs.

La bibliographie concernant les Apoïđea en Roumanie et dans les pays voisins comprend également des références sur la répartition, la fréquence, les époques de vol et les plantes recherchées pour leur nectar et leur pollen; il s'agit en particulier des publications de HENRICH (I880-I884), MOCSARy (I879), SZILADY (I9I4), MÜLLER (I930), Pillich (I935), Moczar (I954), Osichiniuk (I957, I959), etc.

En recherchant les données de notre rapport sur les Apidae Anthophorinae en République Populaive Roumaine (I958), nous avons rassemblé également des observations concernant 1'activité pollinisatrice des Apoïdea. Des références sur la pollinisation de la Luzerne en Hongrie ont été publiées par MoczAR et BöJTös (I957), en Roumanie par IUGA et ScobIor,A (I959).

\section{LE COMPORTEMENT POLLINISATEUR DES APOÏDEA}

Les Apoïdea dépendent des fleurs pour leur nourriture. Le nectar et le pollen sont consommés par les adultes et mis en réserve par les femelles des espèces nidifiantes dans des cellules destinées à cet usage, pour l'alimentation des larves. Les

(1) Oligolectique (oligos, peu; lektos, choisir) : qui ne choisit qu'un petit nombre. Entomol., qui ne butine qu'un petit nombre de plantes ou de fleurs différentes. (Monos, seul; polis, beaucoup). 
mâles sont attirés sur certaines fleurs par le profit nutritif qu'ils en tirent, et aussi par la présence de leurs femelles. Les espèces d'abeilles parasites sont attirées sur les fleurs par une certaine nourriture qui est également celle de leurs hôtes. L'adaptation réciproque des fleurs et de leurs abeilles pollinisatrices a joué un rôle important dans l'évolution et la spécification de ces insectes anthophiles, de même parmi les Angiospermes.

Adoptant la classification de MICHENer (I944), nous avons étudié les groupes suivants parmi les Anthophorinae de la faune roumaine : Anthophorini, Eucerini, Epeolini, Epeoloidini, Melectini, Biastini, Ammobatini, Ammobatoidini, Pasitini. Jusqu'à $195^{8} 8,82$ espèces ont été identifiées, appartenant à I 8 genres de ces 9 groupes d'Anthophorinae. Parmi les genres étudiés 7 nidifient et s'approvisionnent, II sont des parasites. Nous avons particulièrement étudié ces 82 espèces d'Anthophorinae mais nous avons fait également des observations sur les relations entre les fleurs et les autres Apoïdea. Nous nous sommes intéressé également à la pollinisation de la luzerne par les Apoïlea. Bien que nos études sur la pollinisation de diverses plantes cultivées ou spontanées n'aient pas été menées systématiquement, nous pouvons tirer de nos recherches personnelles et des publications concernant la Roumanie ou les pays voisins, quelques conclusions générales.

Nous avons admis que les Apoïlea adultes - mâles et femelles - se nourrissent principalement de pollen à l'époque où ils prennent leur vol. Lorsqu'ils quittent les cellules à couvain, les adultes, qui ont besoin d'albuminoïes pour la maturation de leurs produits génitaux, consomment du pollen. Nous avons observé que les mâles et les femelles de Melitta, Andrena, Meliturga, Tetralonia, etc. doivent ouvrir les fleurs de luzerne puisque du pollen reste fixé sur la partie centrale de la tête. D'autre part, pour maintenir le pourcentage élevé $\left(5^{-7} \mathrm{p}\right.$. roo) d'albuminö̈des dans le sang, les abeilles adultes doivent s'alimenter avec du pollen. Étant donné que le développement des Apoïdea se fait en cellule fermée avec une faible ration de pollen (pincipalement chez les espèces qui prennent leur vol au début du printemps et dont les provisions liquides sont constituées surtout de miel) nous pouvons conclure que l'intense activité des adultes et particulièrement des femelles, n'est possible que grâce à une alimentation protéique.

Chez certains Apoïdea l'activité des mâles sur les fleurs précède de plusieurs jours celle de leurs femelles. Comme ce phénomène apparaît surtout chez des espèces hivernant sous forme d'imagos enfermés dans les cellules à couvain (Anthophora acervorum $\mathrm{L}_{\text {., }} A$. parietina $\mathrm{F}$., Melecta armata PANz.), nous considérons qu'il représente une sélection naturelle et une adaptation aidant à la survie. Ces espèces sortent au clébut du printemps, lorsque les variations climatiques ne permettent que de courtes périodes de vol et que seules quelques plantes sont en fleurs; ce phénomène évite probablement une compétition entre membres de la même espèce. On doit observer que Anthophora acervorum et $A$. parietina abondent en un même lieu et que Melecta armata dépend de ces hôtes pour sa reproduction.

Nous admettons que l'absorption des grains de pollen se fait grâce à un raccourcissement du proboscis. Nous avons signalé (I959) que certaines abeilles à langue courte raccourcissent leur proboscis en repliant dorsalement la glosse dans le canal du prementum. Les Apoïdea à langue longue raccourcissent leur proboscis en repliant ventralement la glosse dont la base s'enroule dans une poche profonde à la partie 
distale du prementum. Au début du printemps, lorsque les espèces hylophiles ( ${ }^{1}$ ) d'Andrena, Osmia, visitent plusieurs sortes d'Ulmus, elles secouent le pollen en heurtant les inflorescences qui restent enveloppées d'auréoles jaunâtres. Les espèces printanières Andrena albicans MUI.L., Osmia rufa L., O. cornuta LATR., visitent Salix et Helleborus car elles se nourrissent de leur pollen. Il est bien connu que certaines fleurs sont recherchées par les abeilles uniquement pour le pollen et d'autres uniquement pour le nectar. Certaines fleurs dépourvues de nectaires (Lysimachia vulgaris I.) sont recherchées par Macropis pour leur pollen et aucune adaptation mutuelle n'est apparue entre la plante et le pollinisateur (Porov, I956). Chez la plupart des races d'Apis mellifera et quelques espèces de Bombus, les ouvrières visitent plusieurs sortes de Medicago, Trifolium et autres légumineuses : elles en absorbent le nectar en introduisant latéralement le proboscis entre la carène et une des ailes. Mais Bombus mastrucatus GERST. qui a un proboscis court, coupe avec ses mandibules le dard des fleurs d'Aconit et atteint la source de nectar à travers l'ouverture. Cependant, les races d'abeilles qui ne recherchent d'ordinaire que le nectar de la luzerne, en récoltent aussi le pollen si l'influence de la température facilite l'ouverture des fleurs ou si celles-ci ont été ouvertes par des Apoïdea sauvages ou des méthodes artificielles (Komarov, I956). La plupart des races d'Apis m. évitent d'ouvrir les fleurs de légumineuses car si elles introduisent leur proboscis au centre de la corolle sans incliner leur tête, comme font les Apoïdea sauvages, il se trouve resserré dans la colonne sexuelle.

Les Apoïdea femelles récoltent le pollen et le nectar pour assurer l'alimentation de leurs larves. Ėn majorité, elles stockent ces produits floraux dans les cellules à couvain préparées spécialement avant la ponte. Les différentes espèces donnent à leurs larves un mélange de pollen et de miel dans des proportions différentes. Lithurgus approvisionne ses cellules à couvain avec du pollen presque sec. Les Andrenidae et les Melittidae humectent le pollen avec du miel de façon à former des pelotes. Les Colletidae et les Andrenidae obtiennent un mélange pâteux ou sirupeux en ajoutant une quantité suffisante de miel. Beaucoup d'Anthophora et de Tetralonia versent une couche semi-liquide de miel sur une sorte de brouet, mais parfois leurs provisions sont presque liquides.

Dans les relations entre les plantes melittophiles et leurs pollinisateurs une distinction a été faite entre les espèces d'insectes polytrophiques $\left({ }^{2}\right)$ (ou euryphages) et oligotrophiques $\left({ }^{3}\right)$ (ou sténophages); il est même question de monolèges, lorsqu'un seul genre de plante (monotypique) $\left({ }^{4}\right)$ fleurit dans certaines régions déterminées pendant le temps de vol, plutôt bref, de l'abeille. Ainsi, Macropis labiata ne visite que Lysimachia vulgaris $\mathrm{L}$. et Colletes succintus $\mathrm{L}$. ne butine que Calluna vulgaris $\mathrm{L}$. Dans certaines régions, Clisodon furcatus PANz. se limite strictement à Stachys, ailleurs il récolte le pollen d'autres Labiatae (Galeopsis, Ballota, Salvia), Borraginaceae (Echium, Anchusa) et Scrophulariaceae (Melampyrum). C. furcatus qui appartient à un ancien genre triasique, émigrant vers le nord. (PoPov, I95I), n'aurait pu

(1) IIylophile (hyle bois ; pheilein, aimer) : qui aime le bois.

(2) Polytrophique (polis, beaucoup ; trophe, nourriture) : dont la nourriture est trìs variée. Syn. euryphage. Anton. monotrophique.

$\left.{ }^{3}\right)$ Oligotrophique (oligos, peu ; trophe, nourriture) : dont la nourriture est peu variée. Anton. eutrophique. Syn. sténophage.

(4) Monotypique (monos, seul; typos, type) : type unique représentant l'espèce. Syn. monolège. Anton. polytypique. 
pénétrer jusqu'aux limites septentrionales de son territoire s'il était devenu monotrophique. Quelques auteurs nomment monolège les espèces qui ne butinent qu'une seule famille de plantes: Tetralonia vicina scabiosae Mocs. se limite aux Composées, selon MoczAR (I954).

Chez beaucoup d'abeilles polytrophiques (Apis, Bombus, Halictus, Andrena, Anthophora, Megachile), une même femelle, même si elle s'approvisionne sur différentes tiges, ne récolte du pollen que sur une sorte de plante au cours d'un ou plusieurs voyages successifs. C'est une caractéristique individuelle, variable même chez les ouvrières de la même famille. Au moyen de méthodes appropriées, toutes les ouvrières d'une famille d'Apis mellifica peuvent être dressées à ne butiner que les plantes dont la pollinisation est jugée nécessaire.

Les Anthophorinae sont en général polytrophiques si 1'on considère leur répartition géographique. Les relations mutuelles entre Apoïdea et plantes melittophiles sont variables car elles se modifient lorsqu'une espèce pénètre dans de nouvelles régions et est soumise à des conditions différentes. Les oligolèges sont nombreux chez les Anthophorinae si l'on considère des aires réduites de leur domaine. Porov (1952, I956) et MrCHENER (I953, I954) ont débattu la relativité du terme oligolectie. LINSI.EY (I958) qualifie d'oligolectiques les espèces dont les individus récoltent le pollen d'une seule espèce de plantes ou d'un groupe d'espèces voisines, même si d'autres sources sont disponibles, utilisant ces dernières uniquement lorsque le pollen spécifique vient à manquer. Nous ne pouvons considérer conme oligotrophiques ces espèces à langue courte (Andrena, Halictus) qui volent au début du printemps, lorsqu'il y a peu de fleurs et dont le butinage se limite à Salix et Tussilago farfara, car elles ne peuvent atteindre les nectaires profonds de Lamium recherchés par Anthophora acervorum $\mathrm{L}$.

Dans la faune de Roumanie et des pays voisins nous considérons comme oligotrophes : Eucera dalmatica I.EP., une espèce sténophage ( $\left.{ }^{1}\right)$ peu répandue qui butine Echium vulgare L. et E. altissimum JACQ., aussi bien en Roumanie qu'en Hongrie ; $E$. excisa MOCz., une espèce sténophage peu répandue mais parfois localement importante, qui butine en Hongrie les Labiées (Salvia nemorosa I; et S. pratensis I.), tandis qu'en Hongrie elle visite les légumineuses (Vicia sativa I; Medicago sativa I.) ; Tetralonia dentata graja FVERSM., une sous-espèce sténophage, qui butine en Hongrie et Roumanie diffétentes sortes de Centaurea mais en Roumanie butine également Carduus nutans L. et Onopordon acanthium L.; se différenciant ainsi de la forme polytrophique $T$. dentata dentata, qui visite les Composées, Labiées et Légumineuses ; $T$. commixa lyncea mocs., une sous-espèce sténophage qui se limite en Roumanie comme en Hongrie à quelques sortes de Carduus; $T$. acutangula dufouri PÉREZ, une sous-espèce sténophage qui recherche en Roumanie aussi bien qu'en Hongrie quelques Composées (Inula germanica L., Senecio erucifolium L., Centaurea cyaneus I., Hieracium) ; T. vicina scabiosae Mocz. qui visite, à la fois en Hongrie et Roumanie les Dipsacacées (Dipsacus, Knautia, Scabiosa) en certains endroits et les Composées (Carduus, Centaurea) en d'autres endroits; Anthophora retusa meridionalis PÉREz, une sous-espèce oligotrophique qui butine en Roumanie Lamium purpureum $\mathrm{L}$. et $L$. album $\mathrm{L}$. à la différence de la forme polytrophique $A$. retusa retusa $\mathrm{L}$., qui visite les Labiées, Borraginacées et Légumineuses. Ayant à notre disposition des données

(1) Sténophage (stenos, étroit phagein, manger) : voir oligotrophique. 
suffisantes concernant les plantes butinées par des types d'insectes jugés de même espèce (I958), nous avons distingué les formes polytrophiques des formes oligotrophiques.

Oligolectie et polylectie ne sont pas des caractéristiques du type de plante que nous avons considéré comme le plus ancien à cause de son aire orientale ou méridionale de répartition. Ainsi, dans le groupe Tetralonia dentata, la forme primitive graja est oligolectique, tandis qu'à l'intérieur du groupe $T$. acutangula la forme dérivée dufouri possède ce caractère. La pénétration d'une espèce dans une nouvelle région s'accompagne souvent d'un changement dans les plantes butinées en rapport avec les plantes qui fleurissent à l'époque du vol et également avec la faune anthophile régionale. Les abeilles qui butinent les Composées ont tendance à devenir oligolectiques.

Les Apoïdea oligotrophiques s'adaptent à certaines plantes melittophiles. Ainsi apparaissent certaines adaptations physiologiques : un temps de vol court correspondant à la floraison des plantes melittophiles; des vols diurnes en rapport avec la disponibilité en pollen (ouverture de fleurs fermées la nuit, évaporation de rosée, etc.). Chez certains de ces insectes, des différences morphologiques facilitent la récolte et le transport du pollen et du nectar de certaines fleurs : les espèces qui butinent les Malvacées ont une brosse à poils longs, épais et soyeux ; celles qui vont chercher le nectar dans les corolles à tube profond, ont un long proboscis, etc. Les Apoïdea oligolectiques acquièrent sans doute également certains réflexes nerveux incontrôlés qui les attirent sur les plantes nourricières lorsqu'elles sont en floraison et dans l'état le plus propice à la récolte du pollen et du nectar. Les abeilles sont attirées par des fleurs qui ont une odeur, une couleur, une forme ou un goût particuliers. Ces réflexes sont dus aux propriétés de la nourriture consommée par l'insecte pendant sa période de croissance. Attirés par l'odeur de la nourriture, les mâles protérandres et quelques femelles d'Andrena volent autour des inflorescences encore fermées de Salix.

Si une espèce d'Apoïdea, par suite d'un isolement géographique, a longtemps vécu dans une région à flore melittophile, les générations successives établissent des relations encore plus étroites et 1'abeille recherche toujours la même plante lorsqu'elle change de territoire. L'oligolectie est utile à la fois pour la plante et pour l'abeille (LINSLEy, I958). Lorsqu'une espèce d'insectes a l'habitude de récolter le pollen d'une ou de plusieurs plantes apparentées, elle devient, pour ces plantes, un pollinisateur plus efficace que celles qui butinent un grand nombre d'espèces végétales.

En devenant oligotrophique, l'abeille réduit ou même annule pendant une période de disette l'effet catastrophique de la lutte pour la vie entre les espèces. Chaque espèce recherchant une nourriture particulière, un certain nombre d'individus parmi la faune apicole de la région aura la chance de survivre et maintiendra ainsi un équilibre naturel. La lutte pour la survie entre les espèces provoque la disparition d'un grand nombre d'individus mais préserve l'existence de l'espèce.

L'abeille polytrophique ayant la possibilité de devenir oligotrophique peut, lorsqu'elle est introduite dans une région nouvelle, se répartir dans l'espace (sur certaines espèces de plantes entomophiles) aussi bien que dans le temps (selon la floraison des plantes respectives). Cette répartition dans l'espace et dans le temps peut mener à une spécificité si, par une sélection naturelle, certaines habitudes s'établissent pendant la période de reproduction, permettant aux individus mieux adaptés 
à une certaine plante et aux conditions climatiques de sa floraison, d'avoir la plus grande progéniture. Parmi ces habitudes peut se trouver l'accouplement sur la plante nourricière. Une fois installés dans une région nouvelle les Apoìdea oligotrophiques peuvent redevenir polytrophiques.

Étant donné la corrélation qui existe entre les plantes melittophiles et leurs pollinisateurs. les Apoïdea sauvages ont un rôle important et indispensable pour le rendement de certaines récoltes : fourrages, pâture, vergers, jardins, etc. Bien que, spécialement en U. R. S. S., des procédés aient été mis au point pour permettre l'utilisation de l'abeille pour accroitre la production par la pollinisation, ils ne sont pas universellement applicables. On a donc appliqué, parallèlement à la pollinisation croisée, certains procédés utilisant les Apoïdea locaux et l'acclimatation de nouvelles espèces.

\section{INVENTAIRE DES POLLINISATEURS DE ROUMANIE}

En Roumanie, où les moyens chimiques de lutte contre les insectes nuisibles n'ont pas encore détruit la faune locale d'Apoïdea et où les procédés d'utilisation de l'abeille (A. mellifera) ne sont pas très répandus, ces pollinisateurs naturels ont un rôle encore important. Il est nécessaire d'étudier les aspects faunistiques : biologie, évolution, survie de ces insectes utiles. On peut tirer des conclusions intéressantes de l'étude des Anthophorinae sur le territoire roumain. L,es 82 espèces étudiées se nourrissent de plantes appartenant à 96 genres et 28 familles: Borraginaceae (I genre), Campanulaceae (2), Caryophylaceae (2), Compositae (I4), Convolvulaceae (I), Cruciferae (2), Dipsacaceae (5), Tiricaceae (I), Fuphorbiaceae (I), Labiatae (I8), Leguminosae (II), Liliaceae (3), Lithraceae (I), Magnoliaceae (I), Malvaceae (3), Oleaceae (I), Papaveraceae (2), Primulaceae (2), Ranunculaceae (2), Resedaceae (2), Rosaceae (4), Salicaceae (I), Scrophulariaceae (2), Solaraceae (I), Umbelliferae (2), Urticaceae (I), Violaceae (I), Apocynaceae (I). Ces Anthophorinae visitent 126 espèces de plantes qui ne sont pas toutes identifiées. Parmi les espèces identifiées, 50 sont mellifères (recherchées par $A$ pis mellifera I.). Les Anthophorinae pollinisent également des plantes introduites dans le pays : Borago officinalis L., Ocimum basilicum L., Majorana hortensis L., Rosmarinus officinalis L., Salvia officinalis L., Medicago sativa L., Pisum sativum L., Robinia pseudaccacia I., Althea rosea L., Hyacinthus orientalis L., Prunus communis L., P. persica L., Lycium halimifolium MiL.., Magnolia. D'autres plantes cultivées, qui poussent spontanément en Roumanie sont aussi pollinisées par les Anthoporinae : Melissa officinalis I., Onobrychis viciaefolia scop., Trifolium pratense L., T. repens L., Vicia sativa L., Syringa vulgaris L., Aquilegia vulgaris L., Malus pumila MrLL., Pyrus piraster I., Prunus avium L., P. cerasus I., Viola tricolor L., Vinca minor L.

Des observations ont montré que la plupart des Anthophorinae sont attirés particulièrement par les Boraginacées : on les trouve sur I dizaine de genres (comprenant I 2 espèces identifiées) parmi les 2 I gentes de la flore roumaine. Ainsi, des 82 espèces d'insectes étudiées, 28 (soit 30,5 p. Ioo) visitent Anchusa et I6 (soit I4 p. I0o) ont été trouvées sur Echium. Anthophora crinipes Sm. a été. trouvé sur des plantes appartenant à la plupart des genres (Anchusa, Borago, Cerinthe, Cynoglossum, Echium, Symphytum); Tetralonia hungarica FRIESE, sur 3 genres (Anchusa, Cerinthe, Nonnea); Eucera tuberculata F. sur 2 genres seulement (Anchusa, Borago). 
Eucera est le genre qui renferme le plus grand nombre d'espèces recherchant Borrago: I2 sur I9 espèces connues en Roumanie. Parmi I5 espèces d'Anthophora identifiées dans notre pays, 9 butinent les Borraginacées, de même que la totalité des espèces (4) d'Amegilla. Ies spécimens des 5 genres (Habropoda, Clisodon, Heliophila, Anthophora, Amegilla) d'Anthophorini de la faune roumaine ont été trouvés sur des plantes appartenant à cette famille qui attire également certaines espèces de Biastini, Ammobatoidini, Epeolini, Melectini, particulièrement Crocisa (4 parmi les 5 espèces reconnues).

Nous avons trouvé des Anthophorinæ sur I4 genres de Composées (comprenant I9 espèces identifiées) seulement parmi les 74 de la flore roumaine. Les plantes de cette famille attirent les abeilles Eucerini : 9 espèces parmi les I4 connues de Tetralonia et 7 parmi les 9 identifiées de Eucera. Les Composées sont butinées également par 3 espèces (sur les 5 trouvées en Roumanie) d'Ammobates, I espèce de Parammobatodes, I de Crocisa, 2 d'Epeolini, Amegilla salviae Mor., Anthophora acervorum L. squalens DRS. et son parasite Melecta armata PANZ.

Les Anthophorinæ butinent également les Labiées : I 8 genres (dont 3 I espèces identifiées) parmi les 30 genres poussant en Roumanie. On trouve la plupart de ces insectes (22 espèces : $26,8 \mathrm{p}$. Ioo) sur Salvia, particulièrement Melecta luctuosa scop. et ses parasites $A$ nthophora parietina $\mathrm{F}$., A. aestivalis PAnz., $A$. retusa $\mathrm{I}$. II espèces butinent Ajuga: I Habropoda, 4 Anthophora 5 Eucera, I Melecta, Io espèces ont été trouvées sur Lamium. I3 espèces butinent Thymus (I5,8 p. IO0), parmi lesquelles $\$$ sont des parasites : Melectini, Biastini, Ammobatini, Pasitini, Epeolini ; leur présence, en grand nombre, sur ces tiges basses et très aromatiques peut s'expliquer par la nécessité de se cacher près du nid de l'hôte creusé dans le sol.

Sur les 3 genres ( 6 espèces identifiées) parmi 7 genres de Malvacées appartenant à la flore roumaine, nous n'avons trouvé que 5 espèces parmi les I 4 connues de $T e$ tralonia. Parmi ces 5 espèces, seule la plus rare $T$. distinguenda MoR. est oligolectique, les autres ( $T$. nana MOR., $T$. pollinosa LEP. $T$. salicariae LEP., $T$. macroglossa II.L. sont polylectiques. Le parasite de cette dernière abeille, Triepeolus tristis SM. a été trouvé également sur Malvaceae.

Nous avons trouvé Anthophorinæ sur II genres de Légumineuses (I5 espèces identifiées), parmi les 40 genres appartenant à la flore roumaine. Parmi les 82 espèces d'insectes étudiées, nous en avons trouvé I5 (I7 p. I0O) sur Trifolium, I2 (I 4,6 p. IOO) sur Vicia, I I (I3,4 p. IOo) sur Coronilla. Le genre qui comprend le plus d'espèces visitant les Leguminosa est Eucera, I3 parmi les I 9 espèces appartiennent à la faune roumaine. Sur les Légumineuses, on trouve également 2 espèces d' $H a$ bropoda, 6 d'Anthophora, 2 d'Amegilla, 5 de Tetralonia, Heliophila bimaculata PAnz., Eupavlovskia funeraria SM., Epeolus variegatus I., Epeoloides coecutiens F.

En dehors des Bourdons, quelques Anthophorinæ sont des pollinisateurs efficaces pour certaines Légumineuses dans les régions qui leur offrent des conditions de vie favorables. Ainsi Trifolium pratense I. est pollinisé par Anthophora aestivalis

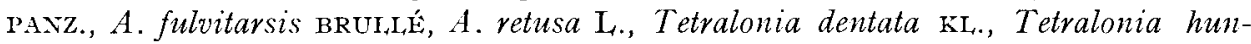
garica FRIESE, T. ruficollis BRUI, É, Eucera tuberculata $\mathrm{F}$. . Nous avons souvent remarqué sur Trifolium repens L.. un grand nombre de Heliophila bimaculata PANz. espèce largement répandue, polytrophique, érémophile $\left(^{1}\right)$ et euryoïque $\left({ }^{2}\right)$.

(1) Érémophile (eremos, solitude : philos, qui aime) : qui aime le désert.

(2) Furyoïgue (eurys, large) : qui a un large biotope. 
Nous avons parfois remarqué que des champs de Pisum sativum L. attirent Eucera interrupta BAER, une espèce polylectique, érémophile et euryoïque.

Sur des champs de Onobrychis viciaefolia scop. nous avons trouvé un grand nombre d'Eucera pollinosa sm., espèce répandue, polylectique, érémophile et sténoïque ${ }^{(1)}$. Nous avons remarqué également, par endroits, sur les mêmes plantes, E. seminuda BRULLÉ, une espèce habituellement peu répandue, polytrophique, érémophile et sténoïque.

Dans certaines régions, les récoltes de Vicia sativa $\mathrm{L}$; sont pollinisées par les espèces suivantes : Eucera clypeata LEP., E. pollinosa SM., E. taurica MoR., E. longicornis L., E. cinerea LEP. Nous avons trouvé aussi sur ces fleurs : $E$. excisa mocs. et $E$. eucnemidea DRs.

Les pollinisateurs efficaces pour les récoltes de Medicago sativa L. sont les Apoïdea à langue longue : Tetralonia dentata $\mathrm{KL} .$, T. hungarica Friese I895, Eucerea cinerea IEP., E. clypeata ER., E. interrupta BAER, Meliturga clavicornis IATR., Megachile argentata F. A Mercurea (district de Sebes), Eucera excisa Mocs., espèce peu commune, oligotrophique, érémophile sténoïque, qui vit là-bas en colonies importantes, a été trouvée sur Medicago sativa L. A Broshteni (district de Focshani) et à Mofleni (district de Craiova), nous avons remarqué sur la luzerne un grand nombre de Eucera eucnemidea DRS., espèce peu commune, oligolectique, étémophile, euryoïque. Dans la steppe ukrainienne, Osichiniuk (I959) a trouvé sur ces plantes fourragères, parmi d'autres Apoïdea, des Anthophorina (7 espèces d'Eucera, I Tetralonia, I Anthophora). Mais les pollinisateurs les plus efficaces des récoltes de luzerne sont les Apoïdea à langue courte, principalement Hylaeus, Colletes, Andrena, Dasypoda, Melitta, Camptopoeum, qui constituaient jusqu'à 76 p. Ioo de 1'ensemble des insectes récoltés. (PoPOV en I95I aboutit aux mêmes conclusions).

Les arbres fruitiers ne dépendent que dans une faible mesure des Anthophorinæ, car l'époque du vol des insectes ne correspond pas avec la floraison des arbres. Cependant les pommiers, poiriers et pruniers attirent quelques espèces hylophiles qui hibernent au stade imago : Anthophora acervorum L. squalens DRs et Melecta armata PANz. sur presque toute l'étendue du pays; $A$. parietina fulvocinerea DRs., $A$. retusa retusa $\mathrm{L}$. et $A$. vulpina PANz. dans les régions de collines. Mais les principaux pollinisateurs des vergers sont, en Roumanie, les espèces précoces d'Osmia, Andrena, Bombus, Colletes.

\section{CONCLUSION}

En raison de leur rôle primordial comme pollinisateurs des récoltes, des plantes fourragères, des pâtures et des vergers, nous considérons que les abeilles sauvages doivent être protégées.

Étant donné que la plupart des Apoïdea vivent soit dans le sol, dans les bois, soit dans les branchages (Fraxinus excelsior L., Acer tataricum L., Prunus spinosa I., Corylus avellana I., etc.), dans les tiges sèches (Verbascum, Rubus, Pencedanum, Arctium, etc.) ou sous les pierres, il est recommandé que les parcelles à semer soient situées au voisinage des bois, des cours d'eau aux berges abruptes, des terrains non

() Sténoique (stenos, étroit) : qui a un biotope étroit. 
arables (Popov, I956). Il faut tenir compte du fait que les Apoïdea préfèrent les endroits ensoleillés et les sols sablonneux et argileux. Il est souhaitable d'élever sur les terrains non irrigués, autour des parcelles à semer, des murettes de terre battue dans lesquelles les Apoïdea peuvent creuser leur nid (PACHINA, I955). Dans les régions où l'on n'élève pas communément des abeilles, l'on devrait implanter autour des vergers des fleurs mellifères (Pulmonaria, Ajuga, Lamium) pour attirer les Apoïdea sauvages sur les arbres fruitiers. Il faudrait éviter de tondre certaines parcelles afin de faciliter l'activité constructrice.

Parmi les mesures générales pour la protection des Apoïdea sauvages doivent être compris les procédés de lutte contre les parasites, prédateurs et pillards. Leur conséquence sur les populations d'Apoïdea varie suivant la région ou les conditions climatiques annuelles. Les prédateurs et parasites sont néfastes surtout pour les larves. Ainsi les Apoidea qui font leur nid en groupe dans le sol ou les branchages (Osmia, Hylaeus, Ceratina, Heriades) sont parfois très décimées.

Reçu pour publication en octobre $\operatorname{Ig}^{6} 5$.

\section{SUMMARY}

THE ANTHOPHILOIS INSECTS OF RUMANIA

The main anthophilous insects of the Carpatho-Danubian region are: Apis mellifica carpatica, Bombus, Halictus, Andrena, Anthophora, Megachile, Eucera, Tetralonia, Osmia and Colletes. 'These insects play a major part in the pollination of crop plants and make an indispensable contribution to the yield of agricultural, horticultural, and forage harvests.

\section{RÉFÉRENCES BIBLIOGRAPHIQUES}

Brschorf II., 1927. Biologie der Hymenopteren. Berlin.

Buagovesicuenskata N. N., 1954. O gnezdovanii pchelinihopilitelei luzerni. Dokl. Akad. Namt S.S.S.R., 99, $859-860$.

DARWI Ch., 1859. On the origin of species by means of nutural sclection. London.

Davidova S. N., 1948. O biologhitsheski osobemosteah $\mathrm{v}$ rabote razlichinih porod phel pri opilenii luzerni. Dokl. Akad. Nauk U.S.S.R., 62, 421-42.3.

IMAvidova S. N., 1956. Medonosnie pcheli i luzema, in : Opilenie selshohosiaistuennih rastenii. Moskva. Filatov F. I., 1945. Vozdelivanie luserni i shiniaca na iugo-vostoke S.S.S.R. Saratov.

Friese H., I923. Die europaïschen Bienen. Das Leben und Wirken unserer Blumeniespen. Berlin.

Grant V., 1949. Pollination Systems as isolating Mechanisms in Angiosperms. Evolution, 3, 82-97.

Gubix A., Kualifman I. I958. Zueti $i$ pchioli, Moskva.

HFineck O., r929. Die Bedeutung der Insekten in Haushalt der Pflanzen, in : Schroeder Ch. Handbuch der Entomologie, Jena, $249^{-} 307$.

IIENRICH C., I 880-I884. Verzeichnis der in Jahre I879-1883 bei Hermannstadt beobachteten Blumenwespen. Verh. Mitl. Siebenb. Ver. Nature. Hernannslall, 1880, 30, 179-182; 1881 31, 68-69; 1882, 32, I 22I $25 ;$ 1 $883, \mathbf{3 3}$, I I 5-1 $6 ;$ I $884, \mathbf{3 4}$, г $3^{6}$.

IugA V. G., I958. Hymenoptera Apoidea, Fam. Apidae, Subfam. Anthophorinae, in : Fauna R.P.R., 9, I-270.

IUGA V. G., I959. Despre principalele apoïde polenizatoare ale lucernelor in : Acad. R.P.R., Omagiu lui Traian Samlescu, Bucuresti, 337-346.

Juraviev A. A., I956. C biologhii tvetenia i opilenia luzernii, in : Opilenie selskohosiaistvennih rastenit, Moskva, $133^{-140 .}$ 
Komakov I. F., 1956. Ob opilenii semennih posevov luzernii pchelami, in : Opilenie selskohosiaistvennih rastenii. Moskva, I44-145.

Linstey li. G., 1958. The Ecology of solitary Bees, Hilgardia, 27, 543-599.

Michener C. D. Life-history Studies in Insect. Systematics. Syst. Zool., 2, I 2-I I8.

Micilener C. D., I958. The Evolution in social Behavior in Bees. Proc. Xth Intern. Congr. Enlomol., $1956,2,44 I-447$.

Michener C. D., Lange R. B., Bigarella J., Salamuxi R., i958. - Factors influencing the Distribution of Bees Nests in Earth Banks. Ecology, 1958, 39, 207-2 I 7 .

Malisinev S. I., I933. SammeIn und Erforschung der Bienen- und Wespennester, in : Abderhalden, Handbuch der Biologischen Arbeitsmethoden, 9, 329-368.

Malishev S. I., I957. Roli pchelinih v proishoshdenii pokritosemennih, Moskva.

Mocsiry A., I879. Data nova ad faunam hymenopterorum Itungariae meridionalis. Acal. hung. scient. Wath. phys. Publ, 16, I-7o.

Mocsar L., Bötjös Z., 1957. A lucenat megporzó méhfélek. Mag. tudom. Akad. Közleményei, 13, $\mathrm{I} 47-\mathrm{I} 78$.

MoCZAR M., I954. Systematik, Verbreitung und Ökologie der Gattungen Eucera LATr. und Telralonia spiv. (Hymenoptera). Ann. hist. nat. Mus. Nat. Hung., 5, 367-386.

Mïlter A., I930. Zur Kenntnis der Insektenfauna der Süddobrudscha. Verh. Mitt. Siebenb. Ver. Nalurw. Ilermtannsiadt., 80, І67-186.

MílLER II., 1873. Die Befruchtung der Blumen durch Insekten und die gegenseitigen Anpassungen beider, Leipzig.

OLesov I. A., 1956. Opit napravlennogo vospitania pchel dlea opilenia luzerni, in Opilenie selskohosiastvennih rastenii, Moskva, I 40-I4I.

Osichisitk A. Z., i957. Novi dlea fauni U.S.S.R. vidibdshil Ak. U.S.S.R. Sbir. pr. zool. Muz., 28, 85-90.

Osicunilk A. Z., I959. Bdiolini (Apoidea) pravobereshnogo stepu Ucraini. Vid Akadem. Nauk U.S.S.R., $\mathrm{I}-90$.

Paxisuor D. V., I956. Dikic nasekomie-opiliteli luzerni, in : Opilenie selskohosiastvennih rastenii, Moskva, 216-2 โ8.

Pacinna L. S., I955. Opiliteli semennoi luzenni v vostochinokasahstanskoi oblasti. Tritdy Inst. Zool. Akadent. Kaz. S.S.R, 4, 226-235.

Piılıch F., I935-1936. Die IIymenopteren fauna Simontornyas. Entontol. Jb, 1935, I I 2-1 22 ; 1936, I33-147.

Popov V. V., I95r. Gheograficheskoie rasprostranenie i evolutsia pchelnih roda. Clisodon. Pat. Zool. Jurn., 243-252.

Porov V. V., 195ı. O znachenii pchelinih (Hymenoptera, Apoidea) pri opilenii luzerni. Trudy Vses. entomol. ob., 43, 65-82.

Popov V. V., i952. O sopreajennih vidah Tridentosmia schured (Hymenoptera, Megachilidae) opiliteleah marevih. Zool. Jurn., 31, 183-190.

Popov V. V., 1952. Pchelinie opiliteli marevih. Zool. Jurn., 31, 494-503.

Porov V. V., r956. Pchelinie, ih sveazi s tsvetcovoi rektitelinostivo i voprosi ob opilenii luzerni. Entom. Obozr., 35, $582-598$.

Stuibrea A. A., I947. Roli medonoknih pchel i dikih v opilenii luzernii. Jurn. Selek. Semen.

SzıLADY Z., I914. Magyarországy rovargyüjtesem jegyrèke, Hymenoptera, Apidae. Rovart. Lap., 21, 78-95.

WheEI ER W. M., 1926. Social life among insects. London. 